\title{
FCNC Decays of the Top Quark
}

\section{Debjyoti Bardhan*†}

Tata Institute of Fundamental Research

E-mail: debjyoti@theory.tifr.res.in

\begin{abstract}
If new physics (e.g. SUSY) does not show up as direct evidence at the LHC, it could still be observable in FCNC processes involving the $t$-quark. We take a close look at the process $t \rightarrow$ $c+h / Z$ and show that its branching ratio in the Standard Model is subject to three mechanisms of suppression. To obtain an observable signal, one needs to evade all these mechanisms in a theory beyond the Standard Model. We show that a theory like the cMSSM cannot provide a big enough enhancement. However, in a framework like $R$-parity-violating SUSY, observable signals are a distinct possibility.
\end{abstract}

9th International Workshop on the CKM Unitarity Triangle

28 November - 3 December 2016

Tata Institute for Fundamental Research (TIFR), Mumbai, India

\footnotetext{
* Speaker.

${ }^{\dagger}$ The speaker wishes to thank all the other authors on the paper. He would especially like to thank Prof. Sreerup Rauychaudhuri and Dr. Diptimoy Ghosh for their help with this talk.
} 


\section{Introduction}

In the search for New Physics (NP), much effort has been devoted to direct searches. Particles at the LHC can be produced either in pairs, or resonantly, or in associated with a Standard Model (SM) particle. However, the energy reach of the LHC is $\sim 2.5 \mathrm{TeV}$, which is roughly the scale at which a significant number of hard interactions take place. If direct searches fail, it makes sense to take a look at indirect searches and exploring Flavour Changing Neutral Current (FCNC) decays of the top quark is one avenue.

The top quark is the heaviest fundamental particle discovered and, unlike other quarks in the SM, it decays before hadronising. Since phenomena like hadronisation are inherently low energy strong coupling effects and an accurate measure of them isn't known, the decay of the top quark, being devoid of these effects, is theoretically clean.

The top quark decays into a $b$-quark and a $W$-boson most of the time, but very rarely also decays into an up-type quark, like a $c$ or a $u$-quark, associated with a neutral boson like a $Z$ or a Higgs boson. These rare decay modes have very small SM branching ratios (BRs) $\left(\sim 10^{-15}\right)$ and are beyond the detection capabilities of the LHC, which can optimistically probe a maximum of $10^{-5}$. We can study the different methods of suppression because of which the BR in the SM is so small; consequently, we can think about a scenario by which the BR can be enhanced. The enhanced BR can be detected at the LHC especially because the SM BR being so small won't swamp out the signal.

\section{Modes of Suppression}

Flavour changing neutral current (FCNC) decays of the top quark are extremely suppressed in the SM and any deviation from this may be identified in colliders as signatures for New Physics. We performed a study of such FCNC decays in [1].

The different mechanisms of suppression that lead to the tiny branching ratios of these FCNC processes are:

- The GIM suppression in these decays

- The Minimal Flavour Violation (MFV) framework which leads to a hierarchy of the values of the CKM matrix elements

- The smallness of the weak coupling constant

In order to study the effects of these modes of suppression in detail, consider a toy model where, apart from the quarks and the Higgs $(H)$, there is a flavour changing charged scalar field $(\omega)$ and the coupling strength between $\omega$ and $H$ is given by $\xi$. The interaction Lagrangian is given by:

$$
\mathscr{L}_{i n t}=\xi \omega^{+} \omega^{-} H+\sum_{i, j=1}^{3}\left(\eta V_{i j} \bar{u}_{i L} d_{j R} \omega^{+}+\text {h.c. }\right)
$$


A similar Lagrangian can be written down for a theory with a $Z$ boson, instead of the Higgs. This is a theoretical laboratory for studying the effects of relaxing each mode of suppression, one at a time. The effect on the amplitude is summarised in Fig. 1.
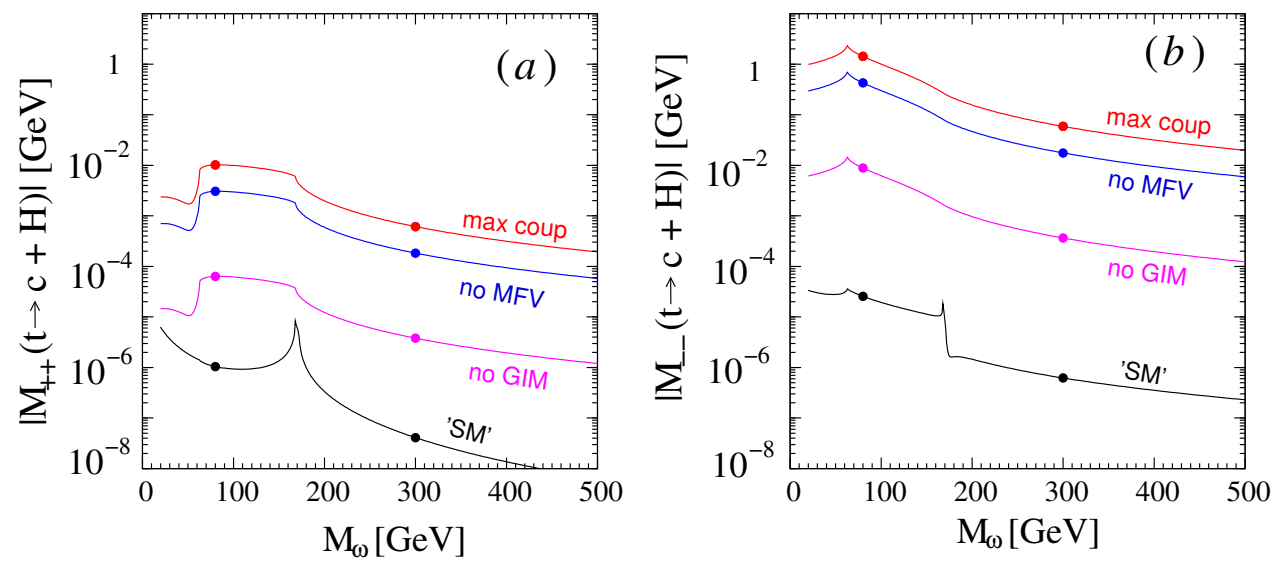

Figure 1: The absolute value of the two helicity amplitudes and how the relaxation of the different suppression factors enhances the amplitude. The subscripts of $\mathscr{M}$, plotted on the y-axis, denotes the helicity of the charm and the top.

The effects on the amplitude can be summarised as follows:

- GIM Mechanism: The unitarity of the CKM matrix leads to this mode of suppression, first shown in [2]. The matrix element for the process for a process in which one quark $q$ changes to another quark $q^{\prime}$ of the same charge can be written as

$$
M_{q q^{\prime}}=\sum_{i=1}^{3} V_{q i}^{*} V_{q^{\prime} i} A\left(x_{i}, M_{W}\right)=\sum_{i=1}^{3} \lambda_{i} A\left(x_{i}, M_{W}\right)
$$

where $x_{i}=m_{i}^{2} / M_{W}^{2}$ carries the generation information, $M_{W}$ is the scale of the charged current interaction and $\lambda_{i}=V_{q i}^{*} V_{q^{\prime} i}$. This can be expanded as

$$
A\left(x_{i}, M_{W}\right)=A_{0}\left(M_{W}\right)+x_{i} A_{i}^{\prime}\left(M_{W}\right)+\frac{1}{2} x_{i}^{2} A_{i}^{\prime \prime}\left(M_{W}\right)+\ldots
$$

where primes represent differentiation with respect to $x_{i}$. Putting this back in 2.2 and using the fact that $\sum_{i} \lambda_{i}=0$, due to the unitarity of the CKM matrix, it can be seen that the dominant term is actually not proportional to $A_{0}$, but to $x_{i} A_{i}^{\prime}$. This gives us a suppression of $x_{i}$ in the amplitude; for the top to charm decay, $x_{i} \sim x_{b} \sim m_{b}^{2} / M_{W}^{2} \sim 10^{-3}$, presenting a suppression of $\sim 10^{-6}$ in the decay width.

- MFV Framework: The MFV framework [3] means that the CKM matrix has a strong hierarchy with regards to the value of its elements. In our case, of a top decay to a charm, the dominant CKM matrix element involved is $V_{t b}^{*} V_{c b} \sim 0.04$. An alternative prescription, which preserves unitarity but doesn't have thehierarchy seen here, can be constructed. For example, we might have

$$
V=\left(\begin{array}{ccc}
1 & 0 & 0 \\
0 & \cos \theta & \sin \theta \\
0 & -\sin \theta & \cos \theta
\end{array}\right)
$$



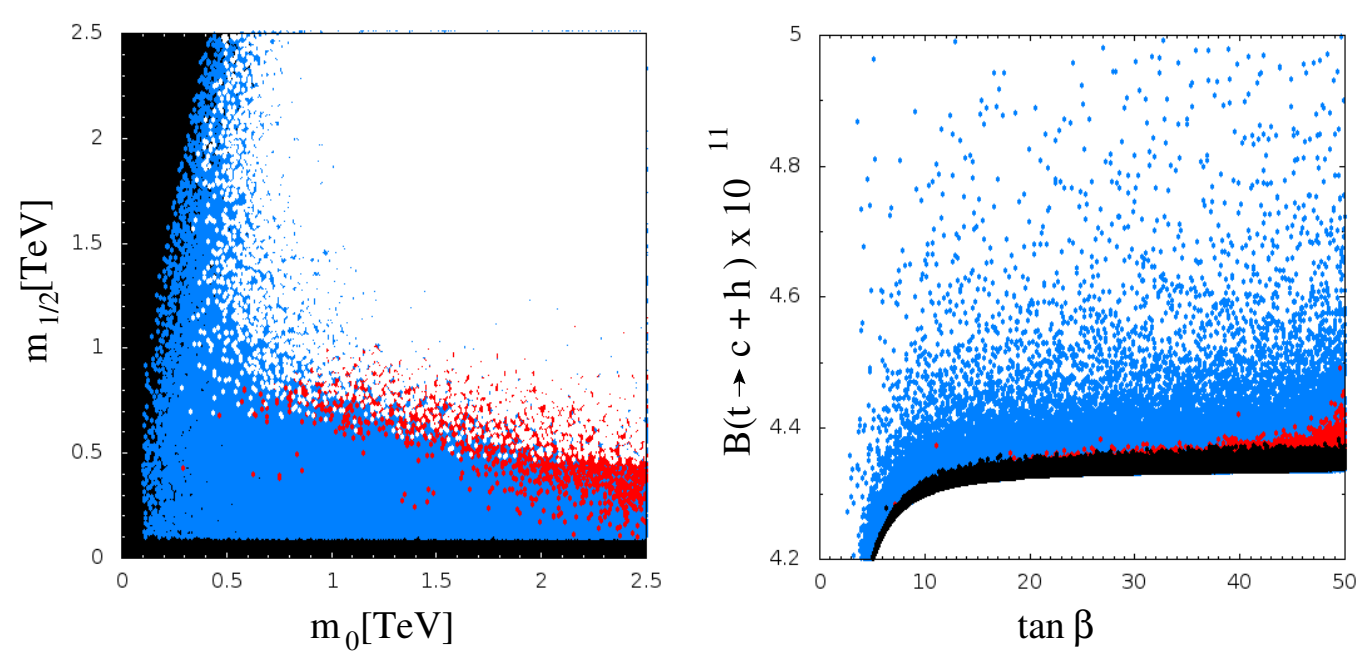

Figure 2: The cMSSM parameter space spanned by $m_{1 / 2}-m_{0}$. The black points are excluded from theoretical considerations, like unstable vacuum, tachyonic states etc. The blue points are excluded by the Higgs mass constraint, while the red points are excluded from flavour constraints. The plot on the right is the branching ratio for these points, where the colours are the same, except that the white (allowed) points on the left correspond to the black points on the right.

The dominant CKM element here is $\lambda_{3}=\sin \theta \cos \theta=0.5 \sin 2 \theta$, which can take up a maximum value of 0.5 , as opposed to the 0.04 as in the previous case.

- Coupling Constant: Finally, the weak coupling constant is rather small in magnitude and we can think upon a new physics model which can have large couplings. In our toy model, we can get a modest enhancement of $\sim 7$.

The overall enhancement in the amplitude is then $10^{3} \times 25 \times 7 \sim 1.75 \times 10^{5}$ and thus, in the branching ratio, it is a factor of $\sim 3 \times 10^{10}$.

\section{Results in different models}

\subsection{Standard Model}

The SM BR is calculated to be $5 \times 10^{-15}$. Violating all the three suppression mechanisms, one can hope to reach a BR of $10^{-5}$.

\section{2 cMSSM}

In the constrained Minimal SuperSymmetric Model (cMSSM), given by the four parameters - the universal fermion mass scale at high energy $m_{1 / 2}$, the universal scalar mass scale at high energy $m_{0}$, the trilinear Higgs coupling parameter $A_{0}$ and the sign of the higgsino mass parameter $\operatorname{sgn}(\mu)$ - there exist charged Higgs bosons which naturally violate the GIM mechanism. 

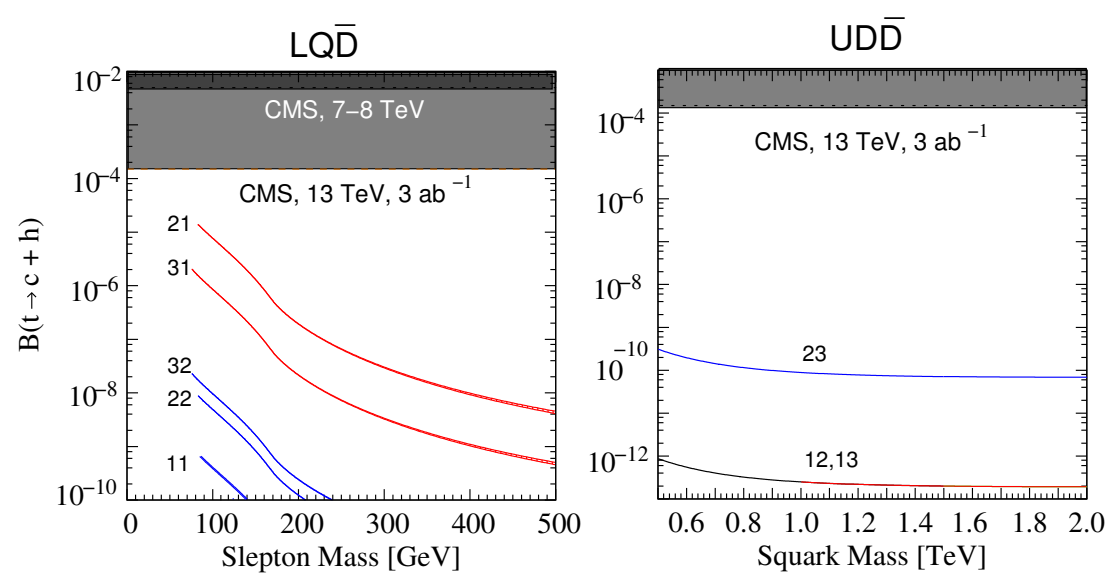

$L Q \bar{D}$
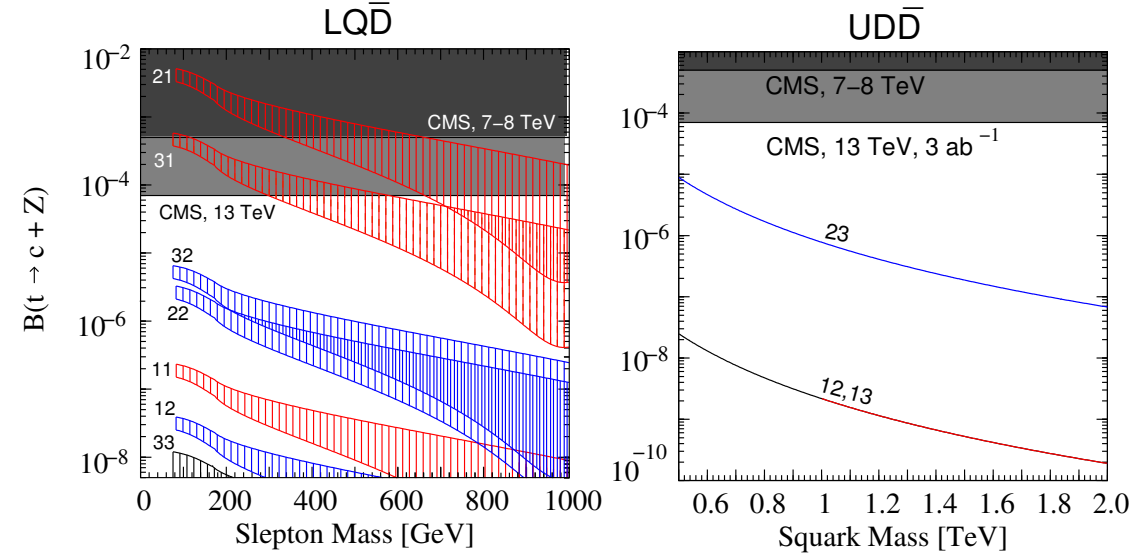

Figure 3: Illustrating the variation in the branching ratios $B\left(t \rightarrow c+h_{0}\right)$ (upper panels) and $B\left(t \rightarrow c+Z_{0}\right)$ (lower panels) with increase in the sfermion masses. For the panels on the left, which show branching ratios proportional to $\left(\lambda_{i 2 k} \lambda_{i 3 k}\right)^{2}$ with the values of ik marked next to each curve, the mass of the slepton $e_{L i}$ is plotted along the abscissa, and the mass of the squark $\tilde{d}_{R k}$ is responsible for the thickness of the lines in the upper panel and the hatched region in the lower panel. For the panels on the right, which show branching ratios proportional to $\left(\lambda_{2 j k} \lambda_{3 j k}\right)^{2}$ with the values of $j k$ marked next to each curve, the mass of the squark $\tilde{d}_{R k}$ is plotted along the abscissa. The dark (light) grey shaded regions represent the experimental bounds (discovery limits) from the LHC, operating at $7-8 \mathrm{TeV}(13 \mathrm{TeV}$, projected

However, the four parameters cannot take any value - they are constrained by various experimental inputs, most notably the Higgs mass. The effect of various constraints can be estimated from Fig 2 .

These constraints are responsible in raising the mass of the charged Higgs bosons in the theory, which suppresses the amplitude significantly. Any enhancement achieved by breaking GIM is offset partially by the heavy charged Higgs in the theory. The cMSSM, furthermore, doesn't go beyond the MFV framework and the enhancement due to the coupling is also not very large. Overall, the cMSSM enhancement doesn't exceed $\sim 10^{4}$ in the branching ratio. 


\subsection{RPV SUSY}

SUSY models which break $R$-parity (a good review of RPV-SUSY is [4]) are particularly interesting in this context for several reasons: firstly, there is no unitary CKM-like mixing matrix, thus there is no GIM suppression; secondly, there is no MFV framework to subscribe to either, and thus there is no hierarchy; finally, several of the $R$-parity violating couplings can be rather large, despite what is commonly understood.

The RPV superpotential is given as:

$$
W_{R_{p}}=\sum_{i, j, k=1}^{3}\left(\frac{1}{2} \lambda_{i j k} \hat{L}_{i} \hat{L}_{j} \hat{E}_{k}^{c}+\lambda_{i j k}^{\prime} \hat{L}_{i} \hat{Q}_{j} \hat{D}_{k}^{c}+\lambda_{i j k}^{\prime \prime} \hat{U}_{i}^{c} \hat{D}_{j}^{c} \hat{D}_{k}^{c}\right)
$$

where each of the fields with a hat $($ on top represents a superfield. $\hat{L}$ and $\hat{Q}$ are $\mathrm{SU}(2)$ superfields, while $\hat{E}, \hat{U}$ and $\hat{D}$ are singlets. We are interested in the $L Q D$ term (second term) and the $U D D$ term (third term) in the Lagrangian. The second term violates lepton number $(\mathrm{L})$ and the third term violates baryon number (B). We can only consider either set of coupling at once, but not both together, as that leads to proton decay.

The results are rather encouraging for the $t \rightarrow c Z$ decays, where we do hit the projected experimental bounds, as shown in Fig. 3

\section{References}

[1] D. Bardhan, G. Bhattacharyya, D. Ghosh, M. Patra and S. Raychaudhuri, Phys. Rev. D94, no. 1, 015026 (2016)

[2] S. L. Glashow, J. Iliopoulos and L. Maiani, Phys. Rev. D2, 1285 (1970).

[3] A. J. Buras, Acta Phys. Polon. B 34, 5615 (2003) [hep-ph/0310208].

[4] R. Barbier et al., Phys. Rept. 420, 1 (2005) 